\title{
ReMauve: A Relational Model Tree Learner
}

\author{
Celine Vens, Jan Ramon, and Hendrik Blockeel \\ Katholieke Universiteit Leuven - Department of Computer Science, \\ Celestijnenlaan 200 A, 3001 Leuven, Belgium \\ \{celine.vens, jan.ramon, hendrik.blockeel\}@cs.kuleuven.be
}

\begin{abstract}
Model trees are a special case of regression trees in which linear regression models are constructed in the leaves. Little attention has been paid to model trees in relational learning, mainly because the task of learning linear regression equations in this context involves dealing with non-determinacy of predictive attributes. Whereas existing approaches handle this non-determinacy issue either by selecting a single value or by aggregating over all values, in this paper we present a model tree learning system that combines both.
\end{abstract}

\section{Introduction}

Model trees are regression trees that contain some non-trivial, usually linear, model in their leaves. In the propositional case, they have been shown to be able to increase predictive performance compared to regression trees that predict the same constant value for each example falling into the same leaf [1-6].

In this paper we investigate the use of model trees in ILP (inductive logic programming, [7]). While classification and regression trees have been around in ILP for several years now [8-10], less can be said about model trees. This may be due to the issues arising when learning a linear regression function in the relational context. Since individuals are related to other objects via one-tomany or many-to-many relationships, the predictive attributes to be included in a regression function may be non-determinate: there may be several instances of them related to the target value. We distinguish a number of approaches to handle non-determinate predictive attributes in regression functions:

1. do not use non-determinate attributes [10]

2. assume one of the instances is relevant

(a) and it can be specified with conditions [11]

(b) and it can not be specified $[12,13]$

3. summarize the instances

(a) using simple aggregate functions defined in advance [14]

(b) using complex aggregate functions

Complex aggregate functions $[15,16]$ are expressed as $\mathcal{F}\left(\sigma_{C}(R)\right)$ in relational algebra, with $\mathcal{F}$ an aggregate function (e.g., exists, max, min, count, ...), $\sigma_{C}(R)$ a selection function based on a condition $C$, and $R$ a set of tuples somehow connected to the tuple we want to classify. They are thus a combination of 
aggregates and selections and therefore approach 3(b) generalizes approaches $2(\mathrm{a})$ and $3(\mathrm{a})$. For example, $\min \{A \mid \operatorname{child}(P, C h)$, age $(C h, A)$, blue_eyes $(C h)\}$ is a complex aggregate which takes the minimum age of a person's children that have blue eyes. In this aggregate, blue_eyes $(C h)$ is the selection condition $C$.

It has been studied how complex aggregates can be efficiently learned to be included in the condition part of a hypothesis [17]. In that work, complex aggregate conditions are learned general-to-specific, subsequently reducing the coverage of the hypothesis. It is still an open problem how complex aggregates can be learned in the conclusion part of a hypothesis (e.g., when the conclusion part is a regression function).

In this paper, we present a model tree system that constructs regression functions with complex aggregates in the leaves. These complex aggregates are not learned at the leaves, but are included in the regression model of a leaf if a linear effect with the target was shown on the path from the root to the leaf. It has been shown $[3,6]$ that model tree learners produce good results if their heuristic function takes linear models into consideration. Most (propositional and relational) systems that use such a heuristic are quadratic [1] or cubic [2, $3,14]$ in the number of numeric attributes. Since we want to include complex aggregates in the search, the number of numeric attributes can become very large, which renders existing systems infeasible to use. Therefore, an important requirement for our system is an efficient heuristic function.

In Sect. 2 we present some related work. Section 3 presents our system in detail. Section 4 presents experimental results. In Sect. 5 we conclude.

\section{Related Work}

The task of relational regression was formalized by Džeroski [18] in the normal ILP framework. This work presents the transformation based system Dinus, which is the first ILP system to address the task of relational regression. The induction is delegated to a propositional learner. Using a model tree learner as RETIS [2], linear regression is used in the model output by Dinus.

Fors [11] is the first system able to predict numbers with non-determinate background knowledge. It is a sequential covering approach that learns rules that contain linear regression models. Non-determinacy among the predictive attributes is handled by testing for the existence of a specific instance giving a number of conditions. If the conditions succeed for more than one instance, the value of the first of these instances is taken.

TILDE [8] and SRT [9] are first order regression tree learners. S-CART [10], the successor of SRT, is capable of including linear models in the leaves. The use of non-determinate predictors in these linear models is not supported. The model trees induced by S-CART are built by first constructing a normal regression tree (using a standard variance reduction heuristic), and afterwards replacing the constant predictions by linear models. This heuristic has been shown to produce sub-optimal model trees in the sense that it tends to split the data set in the wrong places and results in trees that are larger than necessary $[3,6,19]$. 
Appice et al. [14] present a system called MR-SмотI which is a relational upgrade of their propositional Sмоті model tree algorithm [3]. The Sмоті algorithm is different from most model tree inducers in the sense that the multiple linear model that is associated with the leaves is built incrementally from simple linear regression models. These models are introduced by so-called regression nodes occurring in the tree. Each regression node thus adds one term to the multiple regression model and requires updating the target value and other continuous attributes in order to remove the linear effect of the introduced term. To determine the coefficients of a simple linear regression model in a regression node, the problem is locally transformed into a propositional problem by joining the tables from the underlying relational database structure, and normal least squares is applied on this flattened table. Note that this propositionalisation step gives examples that have a higher number of related objects more weight in the least squares procedure. The predicted value for unseen examples is the average prediction for all instances in the propositional representation of the example. Contrary to the efficient methods as S-CART, the systems SMOTI and Mr-Smoti have a high computational complexity. This is due to the heuristic function, which takes into account the fact that linear models are built. It is discussed in more detail further in the paper.

\section{ReMauve}

In this section we present a relational model tree system that is more efficient than Mr-Sмотi, but still uses a heuristic function that takes into account the fact that linear models are constructed at the leaves. Moreover, these models may contain complex aggregates. The system is a relational upgrade of the propositional system Mauve [6], and is called ReMauve (Relational Mauve).

\subsection{Mauve: a propositional model tree learner}

Mauve [6] is a model tree inducer that operates on a single table. It takes as input a number of training examples $e_{i}(i=1 . . n)$ of the form $\left(x_{i 1}, x_{i 2}, \ldots, x_{i m}, y_{i}\right)$, where each $x_{i j}$ denotes the value for the $j$-th independent attribute $X_{j}(j=$ 1..m), and $y_{i}$ is the value for the dependent (target) attribute $Y . Y$ is numeric, while the $X_{j}$ can be numeric or nominal. The system outputs a model tree where each leaf contains a multiple linear regression model that predicts the target in relation to all numeric independent attributes in the table. MAUVE is a TDIDT approach, thus the model tree is built top-down, recursively splitting the training examples according to some condition on the independent attributes.

To estimate the quality of a candidate split, MAUvE proceeds as follows. If the split contains a nominal attribute, the heuristic function is the same as in normal regression trees: the weighted average of the standard deviations in both child nodes, i.e.,

$$
h e u r \_n o m(T)=\frac{\left|E_{l}\right|}{|E|} S D\left(E_{l}\right)+\frac{\left|E_{r}\right|}{|E|} S D\left(E_{r}\right),
$$


where $E$ denotes the set of examples at node $T, E_{l}$ and $E_{r}$ denote the subsets of $E$ associated with the left and right child node of $T$, and $S D\left(E_{m}\right)=$ $\sqrt{\sum_{e_{i} \in E_{m}}\left(y_{i}-\overline{y_{m}}\right)^{2} /\left|E_{m}\right|}$, with $\overline{y_{m}}$ the sample mean of the target attribute in the examples $E_{m}$. If the split concerns a numeric attribute, instead of taking the standard deviation, the residual standard deviation is used, i.e., the root of the mean squared errors calculated w.r.t. a simple linear regression line constructed in the target attribute. The predictive attribute used in the simple regression function is the attribute used in the split. The heuristic of a numeric split at node $T$ is thus

$$
\operatorname{heur\_ num}(T)=\frac{\left|E_{l}\right|}{|E|} R S D\left(E_{l}\right)+\frac{\left|E_{r}\right|}{|E|} R S D\left(E_{r}\right),
$$

with $R S D\left(E_{m}\right)=\sqrt{\sum_{e_{i} \in E_{m}}\left(y_{i}-\left(\alpha_{m} x_{i k}+\beta_{m}\right)\right)^{2} /\left|E_{m}\right|}$, where $\alpha_{m}$ and $\beta_{m}$ are estimated using least squares and $X_{k}$ is the split attribute at $T$, i.e., the split takes the form $X_{k} \leq V$ or $X_{k} \geq V$ with $V$ some value in the domain of $X_{k}$. The split that minimizes this heuristic function (heur_nom $(T)$ or heur_num $(T)$, respectively) is chosen to split $T$.

\subsection{Upgrading Mauve to relational learning}

In this section we discuss how MAUve is upgraded to a relational model tree learner. We first describe the relational regression tree learning system that we start from and afterwards discuss several aspects of the algorithm.

Tilde-RT: a relational regression tree learner. TILDE [8] is a relational top-down induction of decision trees (TDIDT) instantiation, and outputs a first order decision tree, i.e., a decision tree that contains a first order query in the internal nodes. The algorithm is included in the ACE-ilProlog data mining system [20]. TILDE learns both classification and regression trees. The regression tree subsystem is usually denoted by TILDE-RT.

TILDE-RT's procedure to grow a tree is given in Table 1. It takes as input the training examples $E$ and a query $Q$ that corresponds to the empty query. In the recursive calls of the algorithm, $Q$ will represent the conjunction of all succeeding tests from the root of the tree to the node being split. This query will be referred to as the current query. The procedure to grow a node $T$ is as follows. First, a refinement operator generates the set of candidate splits. This set is determined by the language bias given by the user, and by the variables occurring in the current query at $T$. The refinement operator typically operates under $\theta$-subsumption [21] and generates candidates by extending the current query with a number of new literals. Next, the OPTIMAL_SPLIT procedure executes all candidates on the set of examples $E$, estimating the quality of each candidate, and returns the best candidate $Q_{b}$. The quality of a candidate is calculated using a simple heuristic function, similar to MAUVE's heur_nom ${ }^{1}$. The candidate $Q_{b}$

\footnotetext{
${ }^{1}$ In fact, the sum of squared errors is used instead of standard deviation, and an F-test is used to decide whether an improvement is obtained w.r.t. the parent node.
} 
Table 1. TILDE-RT algorithm for first order logical regression tree induction [8].

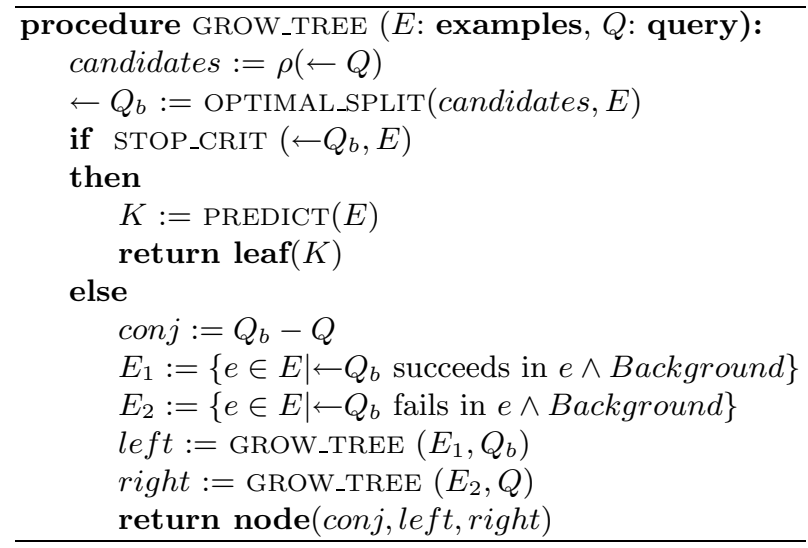

is chosen to split the examples. The conjunction put in the node $T$ consists of $Q_{b}-Q$, i.e., the literals that have been added to $Q$ in order to produce $Q_{b}$. In the left branch, $Q_{b}$ will be further refined, while in the right branch $Q$ is to be refined. When the stop criterion holds (typically, this is when a predefined minimum number of examples is reached), a leaf is built. The PREDICT procedure returns the mean target value of the examples $E$.

Van Assche et al. [16] described how to add (complex) aggregates to the set of candidate splits generated by the refinement operator. This may result in a very large refinement space and it was shown by Vens et al. [17] how the aggregate conditions can be efficiently executed on the examples.

Adapting Tilde-RT's heuristic function. We replaced TILDE-RT's heuristic function by Mauve's heur_nom for nominal, and heur_num for numeric splits.

For the heur_num function, an important issue to deal with concerns the multi-valuedness of the numeric split attribute to be introduced in the regression functions for the $R S D$ calculations. An attribute is determinate if it has exactly one value for each example. It is non-determinate if it may have 0,1 , or more values for each example. For example, the age of a person is determinate, whereas the age of a person's children is non-determinate. Non-determinacy or multivaluedness occurs when an example is related to a set of objects via one-to-many or many-to-many relationships in the relational dataset. In general, two approaches exist to deal with multi-valuedness. ILP systems usually test for the existence of a specific element, thus, a split condition $\operatorname{child}(P, C)$, age $(C, A), A<18$ corresponds to testing the existence of a child with age smaller than 18 . Other approaches [22-24] use aggregate functions (such as max, min, avg, sum,...) to summarize the set of values. The following lemma shows that an ILP test is semantically equivalent to an aggregate function [25]: 
Lemma 1. Let $B$ be a bag of real numbers, and $t$ some real value, then $\exists v \in B: v \geq t$ iff $\max (B) \geq t$, and

$\exists v \in B: v \leq t$ iff $\min (B) \leq t$.

Using this lemma, every numeric attribute results for each example in one deterministic value to feed to the simple linear regression models. The previous nondeterminate numeric attribute would become $\min \{A \mid \operatorname{child}(P, C)$, age $(C, A)\}$.

Adapting Tilde-RT's predictive function. In MAUVE the leaves contain a multiple linear regression function using all numeric attributes as predictors. Adopting this strategy in REMAUVE is not feasible: in relational learning the number of numeric attributes becomes very high, especially when complex aggregate conditions are taken into account. Therefore, we include a numeric attribute in the predictive model of a leaf if it was chosen at a node on the path ${ }^{2}$ from the root to the leaf. The underlying idea is that an attribute would not have been chosen to split the dataset if it did not result in a linear relation with the target in the child nodes.

Dealing with global effects. Consider an attribute that has a global linear effect on the target. Sooner or later in the tree building process this attribute will give rise to a best split, with the same linear effect in both child nodes and will thus generate a superfluous split in the model tree. While the split is redundant, we do want to take into account this attribute in the predictive models at the leaves. Therefore, when the best test for a node $N$ is determined and is found to be a numeric split, the RSD is also calculated for all examples at node $N$. If an F-test considers this RSD equal to the heuristic value of the split, then we know that the linear effect between the split attribute and the target holds in the complete set of examples at $N$, thus it should be introduced in the predictive models in the leaves under $N$ without splitting the data at $N$. To deal with such global linear effects, we introduce unary regression nodes that do not split the data, but only serve to introduce an extra predictor in the linear regression function. The regression nodes contain numeric attributes (without the $>$ or $<$ equation) and pass all examples down to their unique child node. As for split nodes, variables occurring in the attribute of a regression node can be used further down the tree.

In relational learning, especially when aggregates are used, correlation between attributes often comes into play, either true or apparent [26]. For example, in the task of predicting a person's income, the income may increase with the number of children. However, the number of children is correlated with the number of daughters or with the sum of the ages of the children. In our system, if the number of children is an attribute occurring in a regression node, the probability of having an other regression node with the number of daughters is

\footnotetext{
${ }^{2}$ Note that we use the numeric attributes on the complete path from the root to the leaf, not only those from the current query which correspond only to the succeeding tests.
} 
high. To avoid this, the linear effect of numeric attributes occurring in the tree needs to be accounted for. Therefore, after introducing a regression node or a numeric split node, we remove the linear effect of the involved attribute $A$ from the target, i.e., we pass on the residuals $y_{i}-\hat{y}_{i}$ with $\hat{y}_{i}=\alpha * A+\beta$ to the child node(s). In fact, the linear effect should also be removed from all other numeric attributes that can still be used in the model. Given the large number of such attributes, this is not feasible, and instead, when building a regression node $N$ we check whether the involved numeric attribute $A$ has a significant correlation with an attribute in a split or regression node on the path from the root to $N$. If this is the case a leaf is built.

By introducing regression nodes, the analogy with MR-Sмотi increases. A comparison between the two systems is given further in this section.

Stop criterion. We implemented several stop criteria. The first one concerns the minimal number of examples a leaf has to cover. Building a linear model in $k$ attributes in the leaves requires at least $k+1$ examples. Therefore, after refining a node $T$, we check whether each child node of $T$ contains at least $m+1$ examples, where $m$ is the number of numeric attributes occurring on the path from the root to $T$. If this is not the case, $T$ is made a leaf. The second stop criterion calculates the SD of the target values, before they are updated to reflect the linear effect of the best test. If this falls below a certain percentage (default $5 \%$ ) of the original SD at the root node, a leaf is constructed. As a last stop criterion, if the best test turns out to be nominal, an f-test checks whether the corresponding SD value is significantly better than the SD value of the parent node. If not, a leaf node is built. As stated before, for numeric tests, a regression node is built in that case.

The pseudo code of the most important procedures of the algorithm is presented in Table 2.

Undefined attributes. An issue that has not been mentioned in the description of the algorithm is what happens if an attribute is undefined for an example. For example, the age of a person's children is undefined for a person that has no children, or the maximum age of a person's sons is undefined if a person only has daughters. Undefined attributes often occur when using complex aggregates: the selection condition on the set to aggregate over can become so complex that the aggregate is defined only for a few examples. In our system, in order to not violate the monotonicity assumptions assumed by our refinement operator [17], examples for which a split condition is undefined go to the right (failing) branch of the tree. However, this is not sufficient: the heuristic function needs to have a numeric value for each example in the node to be split (also for those going to the right branch) and the linear equations in the leaves need to be able to provide a prediction for each example. Therefore, whenever an explicit value for an undefined attribute is needed (i.e., to calculate the heuristics or to build the 
Table 2. ReMAuve algorithm for first order logical model tree induction.

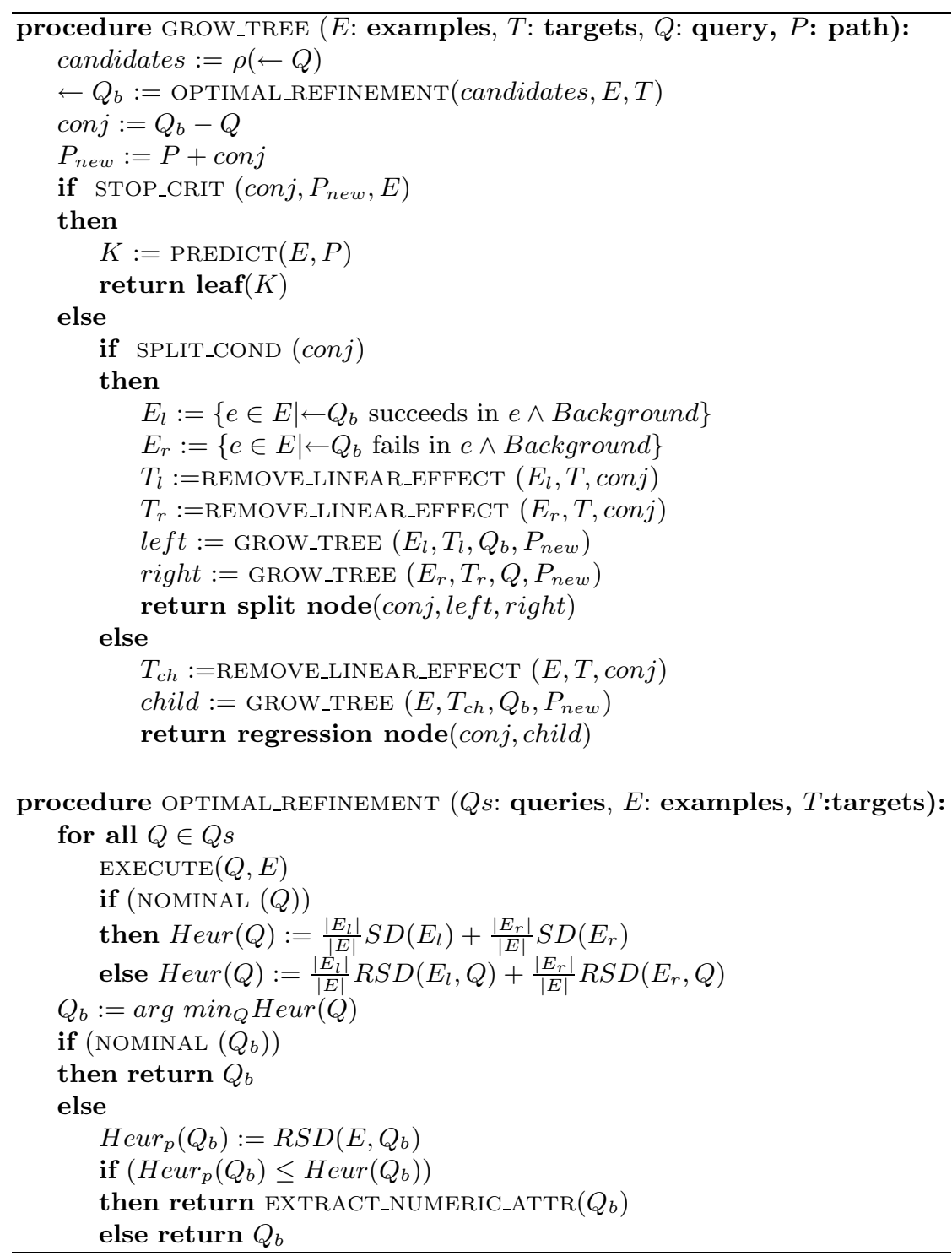


regression functions or make predictions in the leaves), we make use of a default value. There are several possibilities for choosing a default value. We decided to use a value that reduces as much as possible the influence of examples for which the attribute is undefined. The exact values are:

$$
\begin{aligned}
& -F(\emptyset)=\operatorname{avg}\left(F\left(S_{1}\right), F\left(S_{2}\right), \ldots, F\left(S_{n}\right)\right) \text { for } F \in\{\max , \min , \operatorname{avg}, \text { sum }\} \\
& -\operatorname{mode}(\emptyset)=\operatorname{mode}\left(\operatorname{mode}\left(S_{1}\right), \operatorname{mode}\left(S_{2}\right), \ldots, \operatorname{mode}\left(S_{n}\right)\right)
\end{aligned}
$$

where $S_{i}$ is the set of values observed for the attribute for the $i$-th example and $n$ is the number of training examples at the node under consideration for which the attribute is defined.

Comparison with Mr-Smoti. By introducing regression nodes into our system, the resemblance with Mr-Sмоті increases. In the remainder of this section, we discuss the most important differences between both systems.

Complexity of finding the best split node. In REMAUve the evaluation of a numeric split requires the calculation of two simple linear regression functions: one for each child node. In MR-Sмоті a similar, but more complex heuristic function is used: in each child node simple linear regression models are constructed with each numeric attribute used as the predictive attribute. The best regression is chosen independently for the two children and the heuristic value associated with the split under consideration is the weighted average of the RSD of the best regression lines of left and right child. Finding the best numeric split amongst all predictors therefore has complexity $O(m)$ for REMAuve and $O\left(m^{2}\right)$ for MRSMOTI, with $m$ the number of numeric predictors. In the propositional setting of both algorithms the more complex heuristic of SMOTI did not outperform MAUVE on predictive performance [6].

Complexity of introducing regression nodes. In our system, introducing a regression node requires almost no computation: after the best split condition is obtained and is found to be numeric, the global linear effect of the attribute in the split is tested. This requires only one extra RSD to be computed. In MRSMOTI the best regression node is searched for independently of the best split node and requires a lookahead step, in the sense that the best split is searched after the new attribute is included in the multiple model. This renders the whole node selection procedure for MR-SMOTI cubic in the number of predictors.

Removing the linear effect of attributes. In MR-SMOTI regression nodes were introduced in order to incrementally build the multiple regression models in the leaves of the model tree. Therefore, next to updating the target values, the linear effect of an introduced numeric attribute also has to be removed from all other numeric predictors that may be used later in the tree. In REMAUVE it is not possible to update all numeric attributes in the dataset, because these attributes are generated on-the-fly at each node. It would not be feasible to do this updating during refinement generation (requiring another RSD calculation 
for each refinement and each numeric attribute on the path from the root to the node) given the huge search spaces that may be dealt with by introducing complex aggregates. Therefore, in REMAUVE, the final multiple regression model in the leaves is built from scratch.

Overall complexity. The observations above lead to the following overall complexity results. For MR-Sмот, the inner node refinement procedure has complexity $O\left(\mathrm{~m}^{3}\right)$, with $m$ the number of numeric attributes. In a leaf, however, the predictive regression model is obtained by composing the models on the path from the root to the leaf, and thus, can be performed in constant time. A model tree with $k$ inner nodes contains at most $k+1$ leaves, thus the overall complexity for building a model tree with MR-Smoti is $k \times O\left(m^{3}\right)+(k+1) \times O(1)$.

For ReMauve the node refinement process has complexity $O(m)$. Constructing a leaf requires $O\left(p^{3}\right)$, where $p$ is the number of numeric attributes on the path from the root to the leaf. This results in an overall complexity of $k \times O(m)+(k+1) \times O\left(p^{3}\right)$. Given the fact that $p<<m$, especially when using complex aggregates, the REMAUVE system is more efficient for the applications we target.

Representational formalism. A last important difference between both systems concerns their representational formalism. Whereas REMAUVE is an ILP system, MR-Sмоті operates on a relational database, using selection graphs [27] to represent nodes of the model tree.

\section{Experiments}

In this experiments section, we address two questions:

1. How do model trees that predict functions with complex aggregates perform compared to model trees that do not predict aggregates?

2. How does ReMauve compare to other systems as Tilde-RT or MR-Smoti?

We have performed experiments on two biological datasets: Mutagenesis [28] and MassSpectrogram [29]. Given the scarceness of publicly available relational regression datasets with numeric attributes, we also constructed two synthetic datasets.

In Mutagenesis, the task is to predict the mutagenicity level of 230 nitroaromatic compounds. Of these 230 compounds, 188 are known to be well predicted by linear regression methods. In our experiments we use both the regression friendly subset and the full dataset. Several descriptions of the compounds have been proposed [30]. We use the backgrounds B2 (atoms and bonds, including partial charge of atoms) and B3 (B2 extended with the Lumo and $\log P$ properties). In the MassSpectrogram dataset, the task is to predict the weight of a molecule based on its mass spectrogram. A mass spectrogram is a graph of the mass-to-charge ratio of the different fragments versus the frequency. The dataset contains 873 molecules. 
For the synthetic datasets the true target function is a model tree that contains aggregates. They both contain 1000 examples. The first dataset (Artificial1) contains two predictive attributes: $x(X)$ (determinate) and $y(Y)$ (nondeterminate). Each example contains $8 y$ literals, for which the value can be aggregated. All numeric values are random values, uniformly distributed between 0 and 10. The target function for this dataset is shown in Fig. 1(a). It requires two regression nodes in REMAUVE. The second dataset (Artificial2) includes three predictive attributes: $x(X), y(C, Y)$, and $z(Z)$, of which $y$ is non-determinate and has 15 values for each example. Again the numeric values for $x, y$, and $z$ are uniformly distributed between 0 and 10 . The $C$ variable in the $y$ literal is a boolean value. The target function is shown in Fig. 1(b). This dataset also requires two regression nodes, one of which involves a complex aggregate using the boolean condition. For the two datasets, we added Gaussian distributed noise to the target value.

As explained in Sect. 3, ReMAuve is able to learn complex aggregate conditions. In order to address the first question defined above and to allow for a comparison with Mr-Smoti, we also performed the experiments without the ability to learn aggregates.

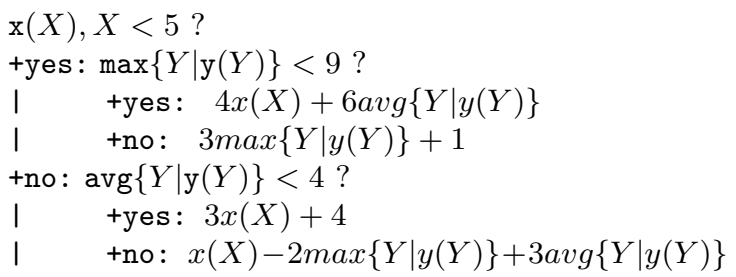

(a)

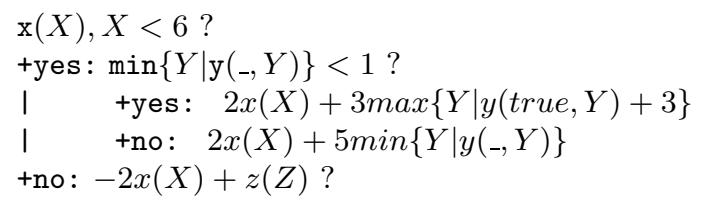

(b)

Fig. 1. Target function for two synthetic datasets. (a) The Artificial1 dataset. (b) The Artificial2 dataset.

The results are presented in Tables 3 and 4 . Predictive performance is obtained by taking the average MSE (mean squared error) of five tenfold crossvalidations. Model size is measured as the number of leaves and the number of regression nodes (the latter only for REMAUVE and MR-Smoti). Induction 
times are difficult to compare, since TILDE-RT and Remauve were run on a different platform than MR-SMOTI.

The first question is dealt with by comparing ReMAuvE's predictive performance when learning complex aggregates to when not learning them. For MassSpectrogram and the artificial datasets, a clear predictive performance improvement is obtained when complex aggregates are considered. Moreover, the improvement holds for both ReMauve and Tilde-RT. Part of the resulting tree for MassSpectrogram is shown in Fig. 2. For Mutagenesis, the result is less obvious. Both for REMAUvE and TILDE-RT the error tends to increase when learning aggregates. Whereas in the classification setting complex aggregates turned out to be beneficial for this task, to our knowledge, complex aggregates have not been used before to predict the numeric mutagenicity level of molecules, thus we can not compare this result to other results in the literature.

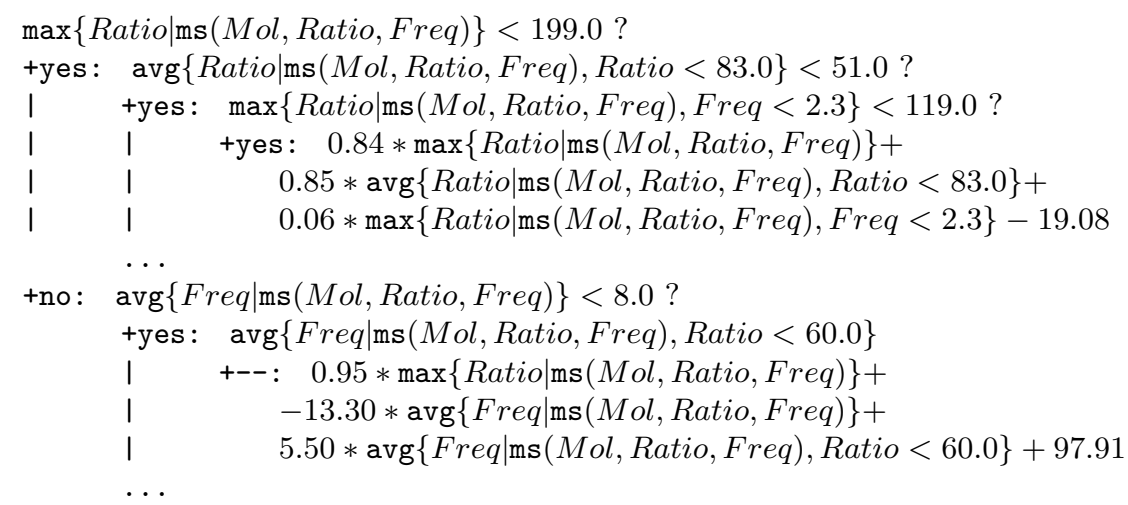

Fig. 2. Resulting tree for the MassSpectrogram dataset.

The second question is answered by comparing ReMauve to TILDE-RT and Mr-Sмоті w.r.t. predictive performance and model complexity. When comparing ReMauve to Tilde-RT, we see that in the aggregate settings (i.e., in the context of many numeric attributes), an improvement in both predictive accuracy and model complexity is obtained. Also, for the artificial datasets, where the target concept involves linear regressions, a clear improvement is obtained, both with and without aggregates. In the other settings, while generally resulting in smaller models, the comparison in predictive performance is less clear. When comparing ReMauve to Mr-Smoti, a first observation is that ReMauve tends to build shorter trees. Only on the Artificial2 dataset is the model built by MR-Sмоті simpler. Regarding predictive performance, we see clear winners for ReMAuve on the artificial datasets. On the MassSpectrogram dataset, MRSmoti outperforms ReMauve. However, when learning complex aggregates, ReMauve reduces Mr-Smoti's MSE with a factor 3.6. On the Mutagenesis datasets, the results are divided: two winners for each system. (The high MSE of 32.68 for MR-Sмоті on the full dataset with background B2 is due to two 
Table 3. Comparing ReMauve's predictive performance and tree size to TILDE-RT and Mr-Smoti for the Mutagenesis dataset.

\begin{tabular}{|c|c|c|c|c|c|c|}
\hline & \multicolumn{6}{|c|}{ Mutagenesis } \\
\hline & \multicolumn{3}{|c|}{ Regression friendly subset } & \multicolumn{3}{|c|}{ Full dataset } \\
\hline & B2 & B3 & B3 & B2 & B3 & B3 \\
\hline & no agg. & no agg. & agg. & no agg. & no agg. & agg. \\
\hline \multicolumn{7}{|l|}{ Avg. MSE } \\
\hline ReMauve & $1.98(0.1)$ & $1.45(0.5)$ & $1.43(0.4)$ & $4.01(0.2)$ & $3.50(0.6)$ & $3.70(0.6)$ \\
\hline TildE-RT & $1.96(0.1)$ & $1.57(0.1)$ & $1.85(0.2)$ & $3.67(0.2)$ & $3.44(0.2)$ & $3.94(0.4)$ \\
\hline Mr-Sмоті & $3.02(0.1)$ & $1.14(0.2)$ & - & $32.68(28.1)$ & $3.32(0.2)$ & - \\
\hline \multicolumn{7}{|l|}{ Regr. nodes } \\
\hline ReMauve & 1 & 2 & 8 & 2 & 1 & 6 \\
\hline Mr-Sмоті & 8 & 5 & - & 8 & 15 & - \\
\hline \multicolumn{7}{|l|}{ Leaves } \\
\hline ReMauve & 7 & 3 & 5 & 11 & 8 & 5 \\
\hline TildE-RT & 14 & 16 & 28 & 11 & 23 & 28 \\
\hline Mr-Sмоті & 10 & 7 & - & 9 & 15 & - \\
\hline
\end{tabular}

particular test examples. Removing them from the test sets yields an average MSE of $4.79(0.12)$.)

\section{Conclusion}

We have presented a relational model tree learner, REMAUVE, that is able to construct regression functions with complex aggregates in the leaves. These complex aggregates occur in the leaves if they have shown a linear relation with the target during the tree building process. The system uses a heuristic function that takes into account the fact that linear models are built in the leaves, while having a time complexity linear in the number of numeric attributes. This differs only a constant factor with the most efficient heuristics, which have been shown to produce sub-optimal model trees. The efficiency is necessary when considering complex aggregates, since the number of numeric attributes becomes very high.

Experimental results demonstrate that, if many numeric attributes occur in the dataset (e.g., in the context of learning aggregates), our system outperforms normal regression tree learners. When comparing to a model tree learner that uses a more complex heuristic function, the comparison in predictive performance is less obvious, while our system in general produces shorter trees.

\section{Acknowledgements}

CV is supported by the GOA 2003/8 project and by the Fund for Scientific Research (FWO) of Flanders. JR and HB are post-doctoral fellows of the Fund for Scientific Research (FWO) of Flanders. The authors thank Bart Demoen for some useful discussions on implementation details and Pieter-Jan Drouillon for providing us with the MassSpectrogram dataset. 
Table 4. Comparing ReMauve's predictive performance and tree size to TILDE-RT and Mr-Smoti for the MassSpectrogram and artificial datasets.

\begin{tabular}{|c|c|c|c|c|c|c|}
\hline & \multicolumn{2}{|c|}{ MassSpectrogram } & \multicolumn{2}{|c|}{ Artificial1 } & \multicolumn{2}{|c|}{ Artificial2 } \\
\hline & no agg. & agg. & no agg. & agg. & no agg. & agg. \\
\hline \multicolumn{7}{|l|}{ Avg. MSE } \\
\hline ReMauve & $8144(65)$ & $1289(101)$ & $30.84(0.2)$ & $1.08(0.0)$ & $1.64(0.1)$ & $0.97(0.0)$ \\
\hline TILDE-RT & $8132(24)$ & $2401(146)$ & $35.18(0.3)$ & $3.94(0.1)$ & $2.55(0.0)$ & $2.06(0.1)$ \\
\hline MR-Sмоті & $4583(221)$ & - & $60.58(2.0)$ & - & $12.08(0.7)$ & - \\
\hline \multicolumn{7}{|c|}{ Regr. nodes } \\
\hline ReMauve & 1 & 9 & 1 & 2 & 2 & 3 \\
\hline Mr-Sмоті & 6 & - & 10 & - & 0 & - \\
\hline \multicolumn{7}{|l|}{ Leaves } \\
\hline ReMauve & 3 & 10 & 5 & 4 & 3 & 3 \\
\hline TILDE-RT & 3 & 222 & 30 & 58 & 44 & 64 \\
\hline Mr-Sмоті & 8 & - & 14 & - & 3 & - \\
\hline
\end{tabular}

\section{References}

1. Alexander, W., Grimshaw, S.: Treed regression. Journal of Computational and Graphical Statistics 5 (1996) 156-175

2. Karalic, A.: Employing linear regression in regression tree leaves. In: European Conference on Artificial Intelligence. (1992) 440-441

3. Malerba, D., Esposito, F., Ceci, M., Appice, A.: Top-down induction of model trees with regression and splitting nodes. IEEE Transactions on Pattern Analysis and Machine Intelligence 26(5) (2004) 612-625

4. Quinlan, J.: Learning with continuous classes. In: Proc. of the 5th Australian Joint Conference on Artificial Intelligence, World Scientific, Singapore (1992) 343-348

5. Torgo, L.: Functional models for regression tree leaves. In: Proceedings of the 14th International Conference on Machine Learning, Morgan Kaufmann (1997) 385-393

6. Vens, C., Blockeel, H.: A simple regression based heuristic for learning model trees. Intelligent Data Analysis 10(3) (2006) 215-236

7. Muggleton, S., ed.: Inductive Logic Programming. Academic Press (1992)

8. Blockeel, H., De Raedt, L.: Top-down induction of first order logical decision trees. Artificial Intelligence 101(1-2) (1998) 285-297

9. Kramer, S.: Structural regression trees. In: Proceedings of the Thirteenth National Conference on Artificial Intelligence, Cambridge/Menlo Park, AAAI Press/MIT Press (1996) 812-819

10. Kramer, S., Widmer, G.: Inducing classification and regression trees in first order logic. In Džeroski, S., Lavrač, N., eds.: Relational Data Mining. Springer-Verlag (2001) 140-159

11. Karalič, A., Bratko, I.: First order regression. Machine Learning 26 (1997) 147-176

12. Ray, S., Page, D.: Multiple instance regression. In: Proceedings of the 18th International Conference on Machine Learning, Morgan Kaufmann (2001) 425-432

13. Srinivasan, A.: Note on the use of statistical procedures as background predicates in ilp. Technical Report PRG-RR-03-09, Oxford University Computing Lab. (2003)

14. Appice, A., Ceci, M., Malerba, D.: Mining model trees: a multi-relational approach. In Horváth, T., Yamamoto, A., eds.: Proceedings of the 13th International Conference on Inductive Logic Programming. Volume 2835 of Lecture Notes in Artificial Intelligence., Springer-Verlag (2003) 4-21 
15. Knobbe, A., Siebes, A., Marseille, B.: Involving aggregate functions in multirelational search. In: Principles of Data Mining and Knowledge Discovery, Proceedings of the 6th European Conference, Springer-Verlag (2002) 287-298

16. Van Assche, A., Vens, C., Blockeel, H., Džeroski, S.: First order random forests: Learning relational classifiers with complex aggregates. Machine Learning 64(1-3) (2006) 149-182

17. Vens, C., Ramon, J., Blockeel, H.: Refining aggregate conditions in relational learning. In Fürnkranz, J., Scheffer, T., Spiliopoulou, M., eds.: Principles of Data Mining and Knowledge Discovery, Proceedings of the 10th European Conference. Volume 4213 of Lecture Notes in Artificial Intelligence., Springer (2006) 383-394

18. Džeroski, S.: Numerical constraints and learnability in inductive logic programming. PhD thesis, University of Ljubljana (Slovenia) (1995)

19. Torgo, L.: Computationally efficient linear regression trees. In: Proc. of the 8th Conf. of the International Federation of Classification Societies, Springer (2002)

20. Blockeel, H., Dehaspe, L., Ramon, J., Struyf, J., Van Assche, A., Vens, C., Fierens, D.: The ace data mining system: User's manual (2006) http://www.cs.kuleuven.be/ dtai/ACE.

21. Plotkin, G.: A note on inductive generalization. Machine Intelligence 5 (1969) $153-163$

22. Koller, D.: Probabilistic relational models. In: Proceedings of the Ninth International Workshop on Inductive Logic Programming. Volume 1634 of Lecture Notes in Artificial Intelligence., Springer-Verlag (1999) 3-13

23. Neville, J., Jensen, D., Friedland, L., Hay, M.: Learning relational probability trees. In: Proceedings of the 9th ACM SIGKDD International Conference on Knowledge Discovery and Data Mining. (2003)

24. Krogel, M.A., Wrobel, S.: Facets of aggregation approaches to propositionalization. In Horváth, T., Yamamoto, A., eds.: Proceedings of the Work-in-Progress Track at the 13th International Conference on Inductive Logic Programming. (2003) 30-39

25. Knobbe, A., Ho, E.: Numbers in multi-relational data mining. In: Principles of Data Mining and Knowledge Discovery, Proceedings of the 9th European Conference, Springer-Verlag (2005) 544-551

26. Jensen, D., Neville, J., Hay, M.: Avoiding bias when aggregating relational data with degree disparity. In: Proceedings of the 20th International Conference on Machine Learning. (2003)

27. Knobbe, A., Siebes, A., van der Wallen, D.: Multi-relational decision tree induction. In: Proceedings of PKDD-1999 - The Third European Conference on Principles and Practice of Knowledge Discovery in Databases. Volume 1704 of Lecture Notes in Computer Science., Prague, Czech Republic, Springer (1999) 378-383

28. Srinivasan, A., Muggleton, S., Sternberg, M., King, R.: Theories for mutagenicity: A study in first-order and feature-based induction. Artificial Intelligence 85(1,2) (1996) 277-299

29. MassSpectrogram dataset, SDBS, National Institute of Advanced Industrial Science and Technology, Japan http://www.aist.go.jp.

30. Srinivasan, A., Muggleton, S., King, R.: Comparing the use of background knowledge by inductive logic programming systems. In De Raedt, L., ed.: Proc. of the 5 th International Workshop on Inductive Logic Programming, Leuven (1995) 199-230 